\title{
Electrogyration in Organic-Inorganic $\left.\left[\left(\mathrm{CH}_{2} \mathrm{OH}\right)_{3} \mathrm{CNH}_{3}\right)\right] \mathrm{H}_{2} \mathrm{PO}_{4}$ Crystals
}

\author{
${ }^{1}$ Vlokh R., ${ }^{1}$ Mys O. and ${ }^{2}$ Czapla Z. \\ ${ }^{1}$ Institute of Physical Optics, 23 Dragomanov St., 79005 Lviv, Ukraine \\ ${ }^{2}$ Institute of Experimental Physics, Wroclaw University, 9 M. Born Sq., Wroclaw, Poland
}

Received: 08.02.2006

\begin{abstract}
The results of studies for electrogyration effect in $\left.\left[\left(\mathrm{CH}_{2} \mathrm{OH}\right)_{3} \mathrm{CNH}_{3}\right)\right] \mathrm{H}_{2} \mathrm{PO}_{4}$ crystals are presented. The coefficient of electrogyration tensor $\gamma_{63}$ is determined.
\end{abstract}

Key words: $\left.\left[\left(\mathrm{CH}_{2} \mathrm{OH}\right)_{3} \mathrm{CNH}_{3}\right)\right] \mathrm{H}_{2} \mathrm{PO}_{4}$ crystals, electrogyration effect

PACS: 78.20.Ek, 78.20.Jq, 78.20.Ci

\section{Introduction}

It has been recently reported that $\left.\left[\left(\mathrm{CH}_{2} \mathrm{OH}\right)_{3} \mathrm{CNH}_{3}\right)\right] \mathrm{H}_{2} \mathrm{PO}_{4} \quad$ organic-inorganic crystals (abbreviated hereafter as TDP) are good electrooptic materials [1]. Pure TDP crystallizes in a non-centrosymmetric space group [2,3]. According to [4], the space symmetry group of TDP crystals is $P 2_{1}$. The present paper is devoted to studies of electrogyration (EG) properties of the TDP crystals.

EG effect is described by the following relation (see, e.g., [5]):

$$
\Delta g_{i j}=\gamma_{i j k} E_{k},
$$

where $\Delta g_{i j}=\Delta \rho \lambda n / \pi$ denotes the increment of the second-rank axial gyration tensor $g_{i j}$ induced by the electric field, $\gamma_{i j k}$ the third-rank axial EG tensor, $\Delta \rho, \lambda$ and $n$ the increment of specific optical rotation, the wavelength and the refractive index, respectively. In recent years, EG effect has been extensively studied in different materials. At least nanocrystals embedded into a silica xerogel matrix [6] and chiral bent-core molecular systems [7] might be mentioned among those materials. Searching for optical materials with high EG performances is important from the point of view of their possible applications in electrically operated optical isolators, wireless devices for highvoltage measurement, electrooptic switches, modulators, etc. Unfortunately, EG rotation of light polarization plane (often termed as an EG rotatory power) has been so far found to be rather small, thus meaning in practice a higher energy consumption, when compare with the corresponding electrooptic devices. However, the EG effect can exist in both noncentrosymmetric and centrocymmetric crystals, whereas the Pockels effect is only possible in a matter without inversion centre. Such the symmetry limitations cause a notable reduction of number of electrooptic materials that could be efficiently used. Just the well known lithium niobate and tantalate, KDP and some other crystals remain. These reasons justify searching for new efficient materials for controlling light electrically. In this work we study experimentally the EG effect in quite new TDP crystals.

\section{Experimental results}

A sample in the from of parallelepiped, with the faces perpendicular to one of the optic axis that lies in $a b$ plane and perpendicular to $c$ axis, was 
prepared from the bulk single crystals grown from aqueous solution. The biasing field was applied along the $c$ axis. EG rotation was determined, using the methods for measuring optical rotatory power of light (the wavelength $\lambda=632.8 \mathrm{~nm}$ ) propagated along one of the optic axes. As reported by the authors [1], the angle between the optic axes is $2 \mathrm{~V}=64.84^{\circ}$ for $\lambda=632.8 \mathrm{~nm}$. The accuracy of absolute determination of the specific rotation of polarization plane induced by electric field was about $25 \%$. For the wavelength mentioned above, crystallographic axes of the TDP crystals coincide with the axes of optical indicatrix ellipsoid. In such a case the matrix of the third-rank axial EG tensor may be written in the crystallographic frame of reference as

\begin{tabular}{c|ccc} 
& $E_{1}$ & $E_{2}$ & $E_{3}$ \\
\hline$\Delta g_{1}$ & 0 & $\gamma_{12}$ & 0 \\
$\Delta g_{2}$ & 0 & $\gamma_{22}$ & 0 \\
$\Delta g_{3}$ & 0 & $\gamma_{32}$ & 0 \\
$\Delta g_{4}$ & $\gamma_{41}$ & 0 & $\gamma_{43}$ \\
$\Delta g_{5}$ & 0 & $\gamma_{52}$ & 0 \\
$\Delta g_{6}$ & $\gamma_{61}$ & 0 & $\gamma_{63}$
\end{tabular}

If the electric field $E_{3}$ is applied and the light propagates in the $a b$ plane, the relation for the pseudo-scalar gyration parameter is as follows:

$$
\Delta G=\Delta g_{i j} l_{i} l_{j}=2\left(\gamma_{63} E_{3} l_{1} l_{2}+\gamma_{43} l_{3} l_{2}\right),
$$

where $\quad l_{1}=\sin \Theta \cos \varphi, \quad l_{2}=\sin \Theta \sin \varphi \quad$ and $l_{3}=\cos \Theta$. Since we have $\varphi=V=32.42^{\circ}$ and $\Theta=90^{\circ}$, Eq. (3) takes the form

$$
\Delta G=2 \gamma_{63} E_{3} \cos V=1.6882 \gamma_{63} E_{3} .
$$

Finally, the expression for the EG coefficient may stands as

$$
\gamma_{63}=\frac{\Delta \rho \lambda n_{c}}{1.6882 \pi E_{3}}
$$

with $n_{c}=1.5284$ (see [1]). It is worth noticing that we did not observe any changes in the angle between the optic axis due to external electric field.
The increment of the optical rotatory power for the TDP induced by the application of biasing field is represented in Fig. 1. The EG effect is linear, i.e. the sign of the mentioned increment depends upon the sign of the electric field. The value of EG coefficient for the TDP crystals calculated on the basis of Eq. (5) is equal to $\gamma_{63}=(0.35 \pm 0.12) \mathrm{pm} / \mathrm{V}$.

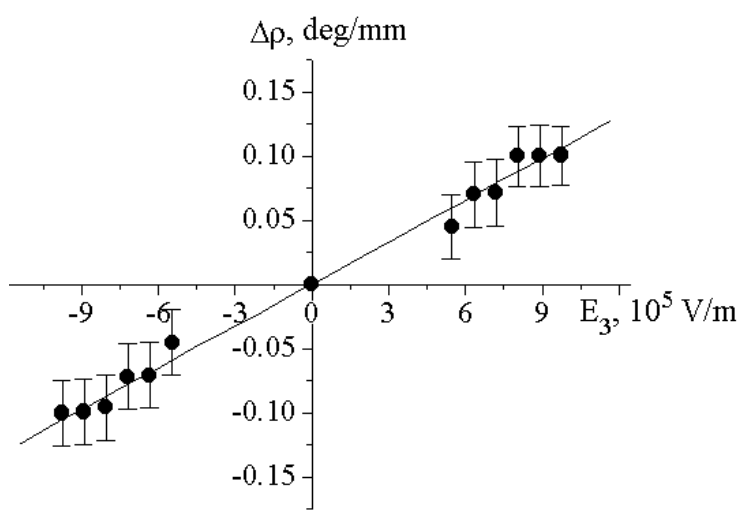

Fig. 1. Increment of optical rotatory power for the TDP crystals observed due to application of biasing electric field ( $T=293 \mathrm{~K}, \lambda=632.8 \mathrm{~nm}$ ).

The EG coefficient for the TDP crystals is comparable with the corresponding parameters detected for the other crystals (see, e.g., [5]). As far as we know, the maximum magnitude of EG coefficients observed at normal conditions, but not in the vicinity of ferroelectric phase transitions, still remains $1.23 \mathrm{pm} / \mathrm{V}$ [8]. It has been reported for $\mathrm{PbMoO}_{4}$ crystals.

\section{Conclusion}

In this work we have studied the EG effect in the TDP crystals. The coefficient of EG tensor has been experimentally determined. It is equal to $\gamma_{63}=(0.35 \pm 0.12) \mathrm{pm} / \mathrm{V}$ at $\mathrm{T}=293 \mathrm{~K}$ and $\lambda=632.8 \mathrm{~nm}$.

\section{Acknowledgement}

The authors from the Institute of Physical Optics are grateful to the Ministry of Education and Science of Ukraine (the Project N0106U000616) for partial financial support of this study. 


\section{References}

1. Vlokh R., Mys O., Romanyuk M., Girnyk I., Martunyuk-Lototska I. and Czapla Z. Ukr. J. Phys. Opt. 6 (2005) 133.

2. Eilerman D. and Rudman R. J. Chem. Phys. 72 (1980) 10.

3. Tamarit J.L., Perez-Jubindo and de la Fuente M.R. J. Phys.: Condens. Matter 9 (1997) 5369.

4. Wu Jingxian, Su Genbo, Huang Oingzhen and Liang Jinkui. Chinese J. Struct. Chem. 7 (1988) 148.
5.Vlokh O.G. Spatial dispersion phenomena in parametric crystal optics. Vyscha shkola, Lviv (1984) 155 p. (in Russian).

6.Kityk I.V., Nyk M., Strek W., Jablonski J.M. and Misiewicz J. J. Phys.: Condens. Matter 17 (2005) 5235.

7. Araoka F., Takanishi Y., Takezoe H., Kim A., Park B. and Wu J. J. Opt. Soc. Am. B 20 (2003) 314.

8. Vlokh O.G., Zheludev I.S. and Klimov I.M. DAN USSR 223 (1975) 1391 (in Russian). 